\title{
Effect of a set of acids and polymerization conditions on the architecture of polycarbonates obtained via ring opening polymerization
}

\author{
Citation for published version (APA): \\ Jiménez-Pardo, I., van der Ven, L. G. J., van Benthem, R. A. T. M., Esteves, A. C. C., \& de With, G. (2017). \\ Effect of a set of acids and polymerization conditions on the architecture of polycarbonates obtained via ring \\ opening polymerization. Journal of Polymer Science, Part A: Polymer Chemistry, 55(9), 1502-1511. \\ https://doi.org/10.1002/pola.28492
}

\section{Document license: \\ TAVERNE}

DOI:

10.1002/pola.28492

Document status and date:

Published: 01/05/2017

\section{Document Version:}

Publisher's PDF, also known as Version of Record (includes final page, issue and volume numbers)

\section{Please check the document version of this publication:}

- A submitted manuscript is the version of the article upon submission and before peer-review. There can be important differences between the submitted version and the official published version of record. People interested in the research are advised to contact the author for the final version of the publication, or visit the $\mathrm{DOI}$ to the publisher's website.

- The final author version and the galley proof are versions of the publication after peer review.

- The final published version features the final layout of the paper including the volume, issue and page numbers.

Link to publication

\footnotetext{
General rights

- You may freely distribute the URL identifying the publication in the public portal. follow below link for the End User Agreement:

www.tue.nl/taverne

\section{Take down policy}

If you believe that this document breaches copyright please contact us at:

openaccess@tue.nl

providing details and we will investigate your claim.
}

Copyright and moral rights for the publications made accessible in the public portal are retained by the authors and/or other copyright owners and it is a condition of accessing publications that users recognise and abide by the legal requirements associated with these rights.

- Users may download and print one copy of any publication from the public portal for the purpose of private study or research.

- You may not further distribute the material or use it for any profit-making activity or commercial gain

If the publication is distributed under the terms of Article $25 \mathrm{fa}$ of the Dutch Copyright Act, indicated by the "Taverne" license above, please 


\title{
Effect of a Set of Acids and Polymerization Conditions on the Architecture of Polycarbonates Obtained via Ring Opening Polymerization
}

\author{
I. Jiménez-Pardo, ${ }^{1}$ L. G. J. van der Ven, ${ }^{1}$ R. A. T. M. van Benthem, ${ }^{1,2}$ \\ A. C. C. Esteves, ${ }^{1}$ G. de With ${ }^{1,3}$ \\ ${ }^{1}$ Laboratory of Physical Chemistry, Department of Chemical Engineering and Chemistry, Eindhoven University of Technology, \\ Eindhoven, The Netherlands \\ ${ }^{2}$ DSM Ahead BV Netherlands, P.O. Box 18, 6160 MD, Geleen, The Netherlands \\ ${ }^{3}$ Laboratory of Materials and Interface Chemistry, Department of Chemical Engineering and Chemistry, Eindhoven University of \\ Technology, Eindhoven, The Netherlands \\ Correspondence to: C. Esteves (E-mail: a.c.c.esteves@tue.nl) or G. de With (E-mail: g.dewith@tue.nl)
}

Received 25 October 2016; accepted 9 December 2016; published online in Wiley Online Library

DOI: $10.1002 /$ pola.28492

ABSTRACT: Polycarbonate-based polymers with a well-defined architecture have become interesting materials due to their large range of applications. Ring opening polymerization (ROP) has been largely applied to make branched polycarbonates. The polymer architectures obtained via this method are strictly related with the polymerization mechanisms involved which depend on the polymerization conditions chosen. Hereby, we evaluate the catalytic activity of three acids, fumaric, trifluoroacetic, and methanesulfonic on the Cationic ROP of trimethylene carbonate (TMC) over a trifunctional initiator, trimethylol propane (TMP), under different reaction conditions. In-detail characterization of the polymers showed the co-existence of two polymerization mechanisms: the activated monomer (AM), which produces a triarmed branched polycarbonate with inclusion of the TMP initiator (TMP-PTMC), and a combined AM/Activated Chain End (ACE) mechanism, which produces a linear polycarbonate (L-PTMC). Such mixtures were identified for nearly all the reaction variables investigated, together with other side reactions. Upon optimization of the synthesis, the polymerizations in toluene with TFA at $35{ }^{\circ} \mathrm{C}$ and equimolar acid/initiator ratio were optimal, avoiding side reactions, but still resulting in a polymer mixture composed of $\sim 69 \%$ TMP-PTMC and $31 \%$ of a polycarbonate linear polymer. The occurrence of such mixed polymer architectures is commonly overlooked in literature regarding CROP of branched polycarbonates. We demonstrate the importance of performing a full characterization for a successful detection of polymer mixtures having different (number of) end-functionalities, which are critical for further use in advanced applications, such as in the biomedical or pharmaceutical filed. (c) 2017 Wiley Periodicals, Inc. J. Polym. Sci., Part A: Polym. Chem. 2017, 55, 1502-1511

KEYWORDS: catalysis; cationic ring opening polymerization; fumaric acid; MALDI; methanesulfonic acid; polycarbonates; trifluoroacetic acid
INTRODUCTION During the last years, the growing and large range of applications of polycarbonate-based polymers, varying from food packaging, automotive, ${ }^{1}$ coatings, ${ }^{2,3}$ electronics ${ }^{4}$ to biomedical applications ${ }^{5-7}$ has stimulated the development of new polymerization methods. Synthetic pathways focusing on the production of well-controlled polymers with a defined composition and a variety of architectures, as for example linear or multi-branched, are therefore increasingly demanded.

One of the most used methods for the synthesis of polycarbonates is ring opening polymerization (ROP) of cyclic carbonates, ${ }^{8}$ using an initiator, generally an alcohol, and a wide diversity of catalysts. This versatile polymerization allows access to the desired molecular design by introducing different functional groups present in both, the carbonate monomer (resulting in lateral polymer chain pendant moieties) and the initiator (resulting in different functionalities in the terminal groups). Furthermore, the number of hydroxyl groups and the morphology of the selected initiator will determine whether the final structure in the obtained polymer is linear or multibranched. This versatility is not only limited to the molecular design but also to other parameters, such as the molecular weight or the molecular mass distribution which can be tuned with fairly good control. In addition, hydroxyl end-groups obtained after insertion of the last monomeric unit open up many post-functionalization possibilities.

Additional Supporting Information may be found in the online version of this article.

(C) 2017 Wiley Periodicals, Inc. 
Depending on the type of catalyst used, ROP can be classified as organocatalytic (cationic, anionic, enzymatic) ${ }^{9-13}$ or metalbased. ${ }^{14-16}$ Although many examples of metal catalysis ROP can be found in the literature, the current trend is to move toward greener synthetic procedures due to the rather large challenge to completely remove the metal residues from the obtained polymer, as its presence can be detrimental for some of the applications, in particular in the biomedical field.

For greener alternatives, cationic ROP (CROP) has emerged as an interesting candidate for polycarbonate synthesis. ${ }^{12,17}$ Besides the advantages mentioned above, CROP makes use of acids as catalysts, which can be selected from a large set of acids with different $\mathrm{pKa}$ values, thus with different reactivities. ${ }^{18}$

A drawback of this polymerization method is the need of high purity monomers, especially when larger amounts are used, thereby limiting the polymer production on multigram scale. However, in the last years various monomers with different functionalities have been developed synthetically which allow larger scale production, even if several purification steps are required before use. $3,19,20$

It is well known that in CROP two polymerization mechanisms can coexist: the activated chain end (ACE) and activated monomer (AM) mechanisms. ${ }^{17}$ Furthermore, other side reactions such as intramolecular transesterification or backbiting producing cyclic species, or decarboxylation producing ether linkages, can also occur. ${ }^{3,21,22}$ These events can lead to a mixture of polymers with different molar mass distributions. The occurrence of these two polymerization mechanisms, which are influenced by the use of different acids as catalysts, as well as, several polymerization conditions, has been widely studied by several authors. ${ }^{23-26}$

When linear polycarbonates are targeted, methanesulfonic acid (MSA) or trifluoroacetic acid (TFA) catalysts have been reported to be most effective in reducing side reactions, with final dispersities $(D)$ of about 1.2 in both cases. ${ }^{25,26}$ For branched polycarbonates much less CROP examples can be found, mostly due to the increasing difficulty in inserting the monomer over the multiple hydroxyl branch points of the initiator. For the synthesis of these multi-arm-based polymers, metal-based catalysis has been mostly explored ${ }^{27-30}$ and only a few examples of cationic-catalyzed ROP can be found in literature. ${ }^{31-36}$

Up to date, the large majority of reports addressing the synthesis of branched polycarbonates have shown polymers with average molecular mass very close to the targeted values, as well as, narrow dispersities $(\nexists)$, based on nuclear magnetic resonance (NMR) and gel permeation chromatography (GPC) characterization. However, so far none reports have shown a detailed characterization of the different molecular mass distributions, discussed the influence of the reaction parameters on the final properties of the polymers, or even the possible co-existence of two polymerization mechanisms and their respective consequences on the final polymer products.
This detailed characterization and in-depth investigation of the reaction parameters allows obtaining an accurate description of the polymer molecular structures, determine quantitatively which polymers are present in the final mixtures (e.g., linear and/or branched) and eventually control the type of polymers and end-functionalities obtained. These features are of extreme relevance for advanced applications, for example in the medical and pharmaceutical field for controlled release of active agents, or in the coatings field in which the end-functionalities are further reacted to build-up cross-linked networks and mechanically resistant layers.

In this paper a trifunctional initiator, trimethylol propane (TMP), was chosen to evaluate the occurrence of the two possible polymerization mechanisms, AM and ACE, in the CROP of trimethylene carbonate (TMC), due to the scarcely addressed full characterization of this kind of multi-armed polymers.

We explore and fully characterize the influence of the different acids and polymerization parameters on the final characteristics of the polycarbonate product, that is, molecular mass, dispersity, mass distribution, and polymer architecture. The catalytic activity of two homogeneous catalysts, (TFA, $\mathrm{pKa}=0.23$ ) and (MSA, $\mathrm{pKa}=-1.9$ ), as well as, a heterogeneous catalyst, fumaric acid $(\mathrm{FA}, \mathrm{pKa}=3.02)$, with different $\mathrm{p} K \mathrm{a}$ values and thus different reactivities, was evaluated The polymerization conditions were varied, that is, reactions with solvent or in bulk, different temperatures, various acid/initiator molar ratio, monomer concentration, and monomer purity were used. The final polymer compositions were analyzed by a combination of NMR, GPC, and Matrix-assisted laser desorption/ionization (MALDI). This in-detail characterization offers the possibility to study the occurrence of the two possible polymerization mechanisms, as well as, other side reactions according to the different acids and polymerization conditions used, leading to determination of the optimal catalysts and reaction conditions for a specific polymer product.

\section{EXPERIMENTAL}

\section{Materials and Methods}

1,3-Propanediol, ethyl chloroformate, triethylamine (TEA), trifluoroacetic acid (TFA, 99\% for HPLC), fumaric acid (FA), methanesulfonic acid (MSA, for HPLC), N,N-diisopropylethylamine, and pyridine were purchased from Sigma-Aldrich. Trimethylol propane (TMP) and aluminum oxide 90 active neutral (activity stage I) for column chromatography were purchased from Merck. TMP and FA were dried at $45{ }^{\circ} \mathrm{C}$ during $3 \mathrm{~h}$ before use. All the other chemicals were used as received. Solvents were purchased from Biosolve.

${ }^{1} \mathrm{H}$ NMR or ${ }^{13} \mathrm{C}$ NMR spectra were recorded on a Varian or Bruker spectrometers, operating at $400 / 100 \mathrm{MHz}$ or $500 / 125$ $\mathrm{MHz}$ (Varian Inova). $\mathrm{CDCl}_{3}$ with TMS as an internal standard was used as the solvent. The reactions were followed in time by ${ }^{1} \mathrm{H}$ NMR to determine the conversion of the monomer, by comparing the signals of the monomer at $4.45 \mathrm{ppm}$ to the 
signals of the polymer at 4.30-4.24 ppm. The reactions were stopped when at least $80 \%$ monomer conversion was reached. Fourier transform-infrared (FT-IR) attenuated total reflectance (ATR) spectroscopy was performed on a Varian 3100 FT-IR spectrometer with DTGS detector, collecting an average of 50 scans in the frequency range from 600 to $4000 \mathrm{~cm}^{-1}$. GPC measurements were performed on a Waters Alliance system GPC equipped with a Waters model 1515 pump and a model 2414 Refractive index detector. A set of two columns (SDV 500 Å, PSS, $30 \mathrm{~cm}, 40^{\circ} \mathrm{C}$, and a guard column (SDV $5 \mu \mathrm{m}$, PSS) was used and THF was selected as eluent with a flow of $1 \mathrm{~mL}$ $\min ^{-1}$. The system was calibrated using narrow molecular mass polystyrene standards ranging from 139 to $39,000 \mathrm{~g}$ $\mathrm{mol}^{-1}$. The polymers were dissolved in THF at a concentration of $1 \mathrm{mg} \mathrm{mL}{ }^{-1}$. Matrix-assisted laser desorption/ionization time-of-flight mass spectrometry (MALDI-ToF MS) measurements were performed on a Voyager-DE Pro instrument (Perspective Biosystems, Framingham, MA). The polymers were dissolved in THF at a concentration of $5 \mathrm{mg} \mathrm{mL}^{-1}$. Potassium trifluoroacetate (KTFA) was used as the ionizing agent and trans-2-[3-(4-tert-butylphenyl)-2-methyl-2-porpenylidene] malononitrile (DCTB) was used as matrix. The isotopic distributions were analyzed using DataExplorer Advanced Biosystems.

\section{Trimethylene Carbonate (TMC) Synthesis}

TMC was synthesized as previously reported by Endo et al., ${ }^{19}$ but introducing a different purification step. Ethyl chloroformate $(25 \mathrm{~mL}, 0.262 \mathrm{~mol})$ was added to a solution of 1,3-propanediol (10 g, $0.132 \mathrm{~mol}$ ) dissolved in $500 \mathrm{~mL}$ of tetrahydrofuran (THF), under a dry nitrogen atmosphere. The reaction mixture was cooled with an ice bath and TEA was added dropwise over a period of $30 \mathrm{~min}$. After $2 \mathrm{~h}$ the white precipitate formed, triethylamine hydrochloride, was filtered off through a neutral aluminum oxide column. The filtered solution was then concentrated in a rotavapor and a white solid was precipitated in $200 \mathrm{~mL}$ of diethyl ether and left overnight in the fridge. The white precipitate (TMC) was collected by filtration and washed with diethyl ether $(3 \times$ $20 \mathrm{~mL}$ ). TMC was dried overnight under vacuum at $35{ }^{\circ} \mathrm{C}$ before use. In case the monomer was not use immediately, it was stored under argon inert atmosphere and always re-dried $\left(35{ }^{\circ} \mathrm{C}\right.$ overnight in vacuum) before use. Yield: $54 \%$. ${ }^{1} \mathrm{H}$ NMR $\left(\mathrm{CDCl}_{3}, 400 \mathrm{MHz}, \delta\right.$ in ppm): 2.14 (m, 2H), 4.45 $(\mathrm{t}, 4 \mathrm{H}) .{ }^{13} \mathrm{C} \mathrm{NMR}\left(\mathrm{CDCl}_{3}, 125 \mathrm{MHz}, \delta\right.$ in ppm): 21.3, 67.9, 148.5. ATR FT-IR ( $v$ in $\mathrm{cm}^{-1}$ ): 2954.9 (C-H st), 1728.2 ( $\mathrm{C}=0 \mathrm{st}), 1188.1$ ( $\mathrm{C}-\mathrm{O}$ st as).

\section{TMP-PTMC Synthesis}

The general procedure for the polymerizations when using fumaric acid (FA) as catalyst was as follows: TMC (0.5 g, 4.9 mmol) and TMP (26 mg, $0.2 \mathrm{mmol}$ ) were added to a flask with $5.4 \mathrm{~mL}$ of dry toluene (monomer concentration $0.9 \mathrm{M}$ ) or without solvent for bulk reactions. The mixture was immersed in an oil bath at the selected reaction temperature and left under dry nitrogen flow for $15 \mathrm{~min}$. After this time, FA was added. Then three vacuum-dry nitrogen cycles were applied to the mixture to ensure oxygen and water free conditions. The reaction mixture was left under stirring for the desired reaction time and thereafter directly dissolved in $1.5 \mathrm{~mL}$ of dichloromethane (DCM), when the reaction was done in bulk, or after evaporation of the toluene, when the reaction was done in the presence of a solvent. The turbid solution obtained was filtered with a $0.2 \mu \mathrm{m}$ Teflon filter to remove the FA residues and the filtered solution was precipitated in $80 \mathrm{~mL}$ of diethyl ether. After decantation of the solvent and washing with diethyl ether $(2 \times 20 \mathrm{~mL})$, TMP-PTMC was obtained as a colorless and sticky oil. Typical yields were between 21\% (105 mg) and 42\% (213 mg).

The general procedure for polymerization when using trifluoroacetic acid (TFA) as catalyst was as follows: TMC ( $0.5 \mathrm{~g}, 4.9 \mathrm{mmol}$ ) and TMP (26 $\mathrm{mg}, 0.2 \mathrm{mmol}$ ) where added to a flask with $5.4 \mathrm{~mL}$ of dry toluene (monomer concentration $0.9 \mathrm{M}$ ), or without solvent for bulk reaction. The mixture was immersed in an oil bath at the selected reaction temperature and left under dry nitrogen flow for $15 \mathrm{~min}$. After this time, TFA was added. Then three vacuum-dry nitrogen cycles were done to the mixture to ensure oxygen and water free conditions. The reaction mixture was stirred for the desired reaction time and thereafter, for polymerizations in bulk, $1.5 \mathrm{~mL}$ of DCM and $0.2 \mathrm{~mL}$ of pyridine were added, to neutralize the acid and stop the reaction. For polymerizations in toluene, only pyridine was used. The toluene fraction was evaporated in the rotavapor and the resulting oil was dissolved in $1.5 \mathrm{~mL}$ of DCM. The reaction mixture dissolved in DCM was precipitated in $80 \mathrm{~mL}$ of diethyl ether and the product TMP-PTMC was obtained, after decantation of the solvent and washing with diethyl ether $(2 \times 20 \mathrm{~mL})$, as a colorless and sticky oil. Typical yields were between $45 \%$ (227 mg) and 76\% (386 mg).

The polymerization with a pre-distillation step when using trifluoroacetic acid (TFA) as catalyst was as follows: TMC $(0.5 \mathrm{~g}$, $4.9 \mathrm{mmol}$ ) and TMP (26 mg, $0.2 \mathrm{mmol}$ ) where added to a flask with $5.4 \mathrm{~mL}$ of dry toluene (monomer concentration $0.9 \mathrm{M}$ ) and an additional volume of $20 \mathrm{~mL}$ of dry toluene was added. Thereafter, $20 \mathrm{~mL}$ of toluene were distilled under vacuum and then, TFA was added. Then three vacuum-dry nitrogen cycles were done to the mixture to ensure oxygen and water free conditions. The reaction procedure and purification were the same as described previously for TFA polymerizations.

The general procedure for polymerization when using methanesulfonic acid (MSA) as catalyst was as follows: TMC ( $0.5 \mathrm{~g}, 4.9 \mathrm{mmol}$ ) and TMP (26 $\mathrm{mg}, 0.2 \mathrm{mmol}$ ) were added to a flask with $5.4 \mathrm{~mL}$ of dry toluene (monomer concentration $0.9 \mathrm{M}$ ). The mixture was immersed in an oil bath at $30{ }^{\circ} \mathrm{C}$, left under dry nitrogen flow for $15 \mathrm{~min}$, and thereafter MSA was added. The reaction mixture was stirred for $2.5 \mathrm{~h}$ and thereafter, $0.2 \mathrm{~mL}$ of $\mathrm{N}, \mathrm{N}$-diisopropylethylamine was added, to neutralize the acid and stop the reaction. The toluene fraction was evaporated in a rotavapor and the resulting oil was dissolved in $1.5 \mathrm{~mL}$ of DCM. The reaction mixture dissolved in DCM was precipitated in $80 \mathrm{~mL}$ of methanol and the product TMP-PTMC was obtained, after decantation 

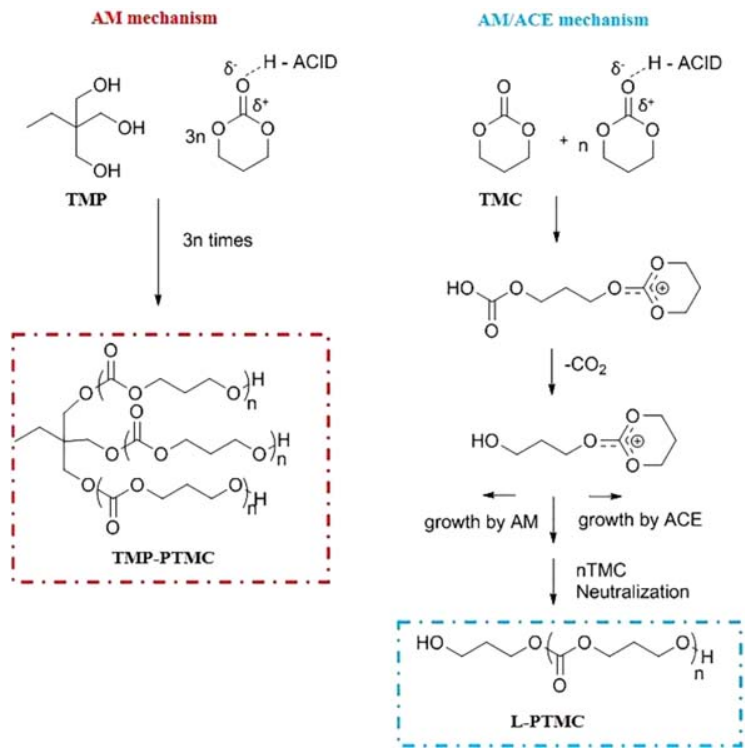

SCHEME 1 ROP of TMC with acid catalyst and initiated by TMP. The polymerization can follow an activated monomer (AM) mechanism, left side path, and/or the AM/Active Chain End (ACE) mechanism, right side path, depending on the acid catalyst and reaction conditions used. Scheme adapted from literature. ${ }^{25}$ [Color figure can be viewed at wileyonlinelibrary. com]

of the solvent and washing with methanol $(2 \times 20 \mathrm{~mL})$, as a colorless and sticky oil. Typical yields were between $45 \%$ (226 mg) and 66\% (334 mg).

TMP-PTMC characterization. ${ }^{1} \mathrm{H}$ NMR $\left(\mathrm{CDCl}_{3}, 400 \mathrm{MHz}, \delta\right.$ in ppm): $0.91(\mathrm{t}), 1.53(\mathrm{~m}), 1.92(\mathrm{~m}), 2.05(\mathrm{~m}), 3.74(\mathrm{t}), 4.11$ (s), 4.30-4.24 (m). ${ }^{13} \mathrm{C} \mathrm{NMR}\left(\mathrm{CDCl}_{3}, 125 \mathrm{MHz}, \delta\right.$ in ppm): 7.25, 21.88, 28.03, 31.63, 42.69, 58.86, 64.30, 64.48, 65.03, $154.79,154.90,155.26$. ATR FT-IR $\left(v\right.$ in $\left.\mathrm{cm}^{-1}\right): 3554.8(\mathrm{OH})$, 2970.4 (C-H st), 1735.9 ( $C=0$ st), 1226.7 ( $C-0$ st as).

\section{RESULTS AND DISCUSSION}

\section{Monomer Synthesis}

The TMC monomer was successfully synthesized by using the method described by Endo et al., ${ }^{19}$ but with a modification of the purification steps. In our monomer synthesis, the original purification process by three recrystallization steps was replaced by a filtration through a short neutral aluminum oxide column. The neutral aluminum oxide column proved to be an effective substrate for the complete retention of triethylamine hydrochloride (see Fig. S1† for comparison of monomer characterization after filter paper filtration and neutral aluminum oxide filtration). Thereafter, the TMC monomer was obtained without detectable signals of the hydrochloride sub-product, as indicated by the solely presence of signals at $4.45 \mathrm{ppm}$ and $2.14 \mathrm{ppm}$ in the ${ }^{1} \mathrm{H}$ NMR spectrum (Figs. S1 and S2†, ${ }^{1} \mathrm{H}$ NMR and ${ }^{13} \mathrm{C}$ NMR, respectively). Moreover, reasonable yields of about 54\% were achieved with a multi-gram scale synthetic procedure, as required for producing the polymer at sufficient large scale.

\section{Polymer Synthesis and Characterization}

The synthetic pathway for making branched polycarbonates via CROP of the 6-membered cyclic carbonate, initiated by the tri-arm alcohol-initiator (TMP) and in the presence of an acid catalyst (from here on named as TMP-PTMC), is based on the activated monomer mechanism (AM), represented in Scheme 1, left path. A parallel Active Chain End (ACE) mechanism can occur, when one acid activated TMC monomeric unit is open by another TMC unit, producing a di-reactive 1,3-propanediol-based intermediate after decarboxylation (Scheme 1, right path). This intermediate can act as bifunctional initiator producing a linear polymer (L-PTMC), which grows further simultaneously by AM and ACE.

To study the selective growth of the polymer by one or both polymerization mechanisms, while avoiding side reactions, we investigated the following reaction variations: type of acidcatalyst, use of solvent in the reaction or polymerization in bulk, temperature, acid to initiator ratio, monomer concentration and monomer purity. The different reaction conditions and the characterization of the polymers obtained are summarized in Table 1.

The polymers obtained were characterized by ${ }^{1} \mathrm{H}$ NMR spectroscopy and GPC to evaluate the initiation step by TMP, the molecular mass (controlled by the monomer/initiator molar ratio) and molar mass dispersity $Ð$. Complementary characterization by MALDI is particularly important to study the composition of the polymers due to the possible coexistence of the two different polymerization mechanisms (according to Scheme 1), which can lead to a mixture of polymer distributions, as explained in the introduction. ${ }^{17}$

A typical ${ }^{1} \mathrm{H}$ NMR spectrum and the respective assignment of the chemical shifts, is shown in Figure 1. Initiation by TMP was confirmed by the presence of the characteristic proton signals for this molecule, one triplet at $0.91 \mathrm{ppm}$ ("a" protons) and a singlet at $4.11 \mathrm{ppm}$ ("c" protons) ("b" protons signals overlapped with the water signal). A poor correlation between the integrated values for the "c" protons of TMP and the "g" protons for the polycarbonate chains was found, that is, the number of "g" protons was higher than expected. This fact was a first indication of the formation of polycarbonate polymers, different from expected theoretically by the AM mechanism only, pointing to the coexistence of two polymerization mechanisms, as discussed later.

Although ${ }^{1} \mathrm{H}$ NMR spectra confirmed the TMP incorporation in the polymer, this characterization only does not provide information about the polymer structure or molecular mass distributions. In the following sections the effect of polymerization conditions, with FA, TFA, or MSA as catalysts, on the polymerization rate, as well as, on the composition of the polymers are compared and discussed with in-detail characterization by MALDI, and focusing on the identification of the polymers produced by the AM and/or ACE mechanisms competing. 
TABLE 1 Polymerization Reactions of TMC with TMP As Initiator and Fumaric Acid (FA), Trifluoroacetic acid (TFA), or Methanesulfonic Acid (MSA) As Catalyst

\begin{tabular}{|c|c|c|c|c|c|c|c|c|}
\hline Run $^{a}$ & Catalyst & Solvent & Temperature $\left({ }^{\circ} \mathrm{C}\right)$ & $A / l^{b}$ & Time (h) & Conversion $^{\mathrm{c}}(\%)$ & $\begin{array}{l}M_{\mathrm{n}}^{\mathrm{GPC}} \\
\left(\mathrm{g} \mathrm{mol}^{-1}\right)\end{array}$ & $D^{\mathrm{GPC}}$ \\
\hline \multirow[t]{2}{*}{1} & FA & Toluene & 90 & 2.5 & 24 & 17 & 3350 & 1.16 \\
\hline & & & & & 120 & 88 & & \\
\hline \multirow[t]{2}{*}{2} & FA & None & 90 & 2.5 & 24 & 45 & 3550 & 1.32 \\
\hline & & & & & 48 & 86 & & \\
\hline \multirow[t]{2}{*}{3} & FA & None & 100 & 2.5 & 24 & 78 & 3750 & 1.27 \\
\hline & & & & & 48 & 98 & & - \\
\hline $4^{d}$ & FA & None & 90 & 2.5 & 72 & 99 & 3350 & 1.30 \\
\hline \multirow[t]{2}{*}{5} & FA & None & 90 & 1 & 24 & 68 & 2050 & 1.14 \\
\hline & & & & & 48 & 96 & & \\
\hline \multirow[t]{2}{*}{6} & FA & None & 90 & 0.5 & 24 & 55 & 2300 & 1.25 \\
\hline & & & & & 48 & 87 & & \\
\hline 7 & TFA & Toluene & 70 & 2.5 & 24 & 94 & 3550 & 1.32 \\
\hline 8 & TFA & Toluene & 50 & 2.5 & 24 & 98 & 3950 & 1.29 \\
\hline 9 & TFA & Toluene & 35 & 2.5 & 24 & 99 & 3700 & 1.20 \\
\hline \multirow[t]{2}{*}{10} & TFA & Toluene & 35 & 1 & 24 & 58 & 2550 & 1.07 \\
\hline & & & & & 48 & 94 & & \\
\hline $11^{e}$ & TFA & Toluene & 35 & 1 & 48 & - & 2200 & 1.11 \\
\hline \multirow[t]{3}{*}{12} & TFA & Toluene & 35 & 0.5 & 24 & 13 & 2200 & 1.13 \\
\hline & & & & & 48 & 43 & & \\
\hline & & & & & 168 & 94 & & \\
\hline 13 & TFA & None & 70 & 2.5 & 24 & 82 & 2450 & 1.17 \\
\hline 14 & TFA & None & 50 & 2.5 & 24 & 96 & 2950 & 1.15 \\
\hline 15 & TFA & None & 35 & 2.5 & 24 & 95 & 2550 & 1.12 \\
\hline 16 & MSA & Toluene & 30 & 2.5 & 2.5 & 100 & 4600 & 1.54 \\
\hline 17 & MSA & Toluene & 30 & 1 & 2.5 & 100 & 4350 & 1.49 \\
\hline 18 & MSA & Toluene & 30 & 0.5 & 2.5 & 100 & 4500 & 1.49 \\
\hline
\end{tabular}

a All reactions were done at monomer/initiator (M/l) mol ratio of 25 , to reach a degree of polymerization of 8 , with a molecular weight of 2582 $\mathrm{g} \mathrm{mol}^{-1}$.

b Acid/initiator mol ratio.

Effect of Polymerization in Solvent or in Bulk

For reactions with FA carried out with solvent or in bulk (run 1 vs. run 2-6 in Table 1) a large influence of the solvent was observed; for the polymerization in the
${ }^{\mathrm{c}}$ Conversion of TMC determined by ${ }^{1} \mathrm{H}$ NMR.

d Run 4: monomer was added in several steps to maintain $M / /$ ratios equal or lower than 10 .

e Run 11: introduction of a pre-distillation step before polymerization.

presence of toluene a longer reaction time was needed to reach high conversion, as compared with the bulk reaction. FA acts as heterogeneous catalyst in both cases. $^{37}$

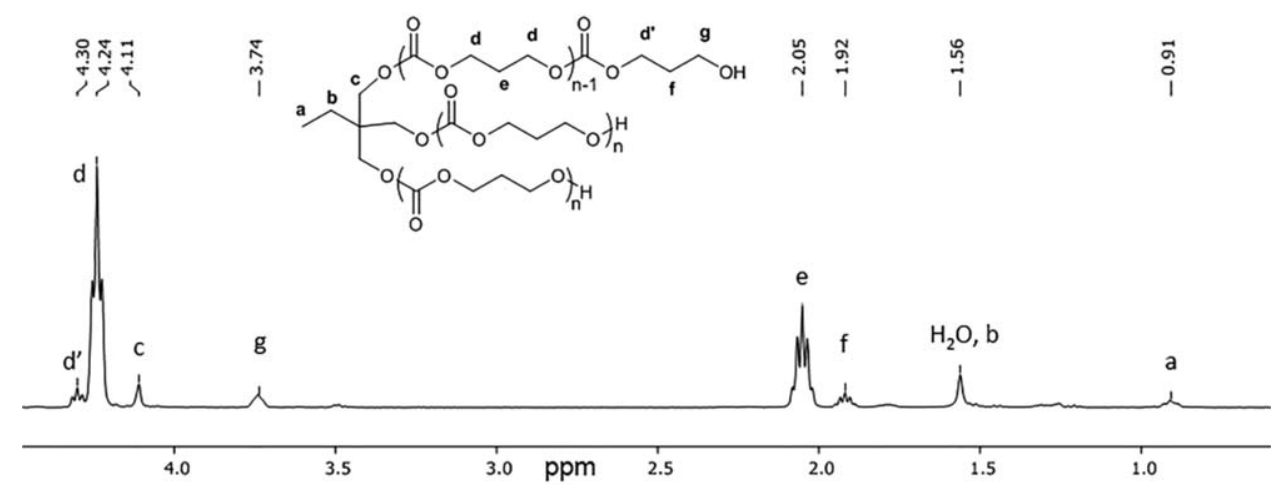

FIGURE 1 Representative ${ }^{1} \mathrm{H}$ NMR spectrum of the polymer product obtained in run 1 in $\mathrm{CDCl}_{3}$. 


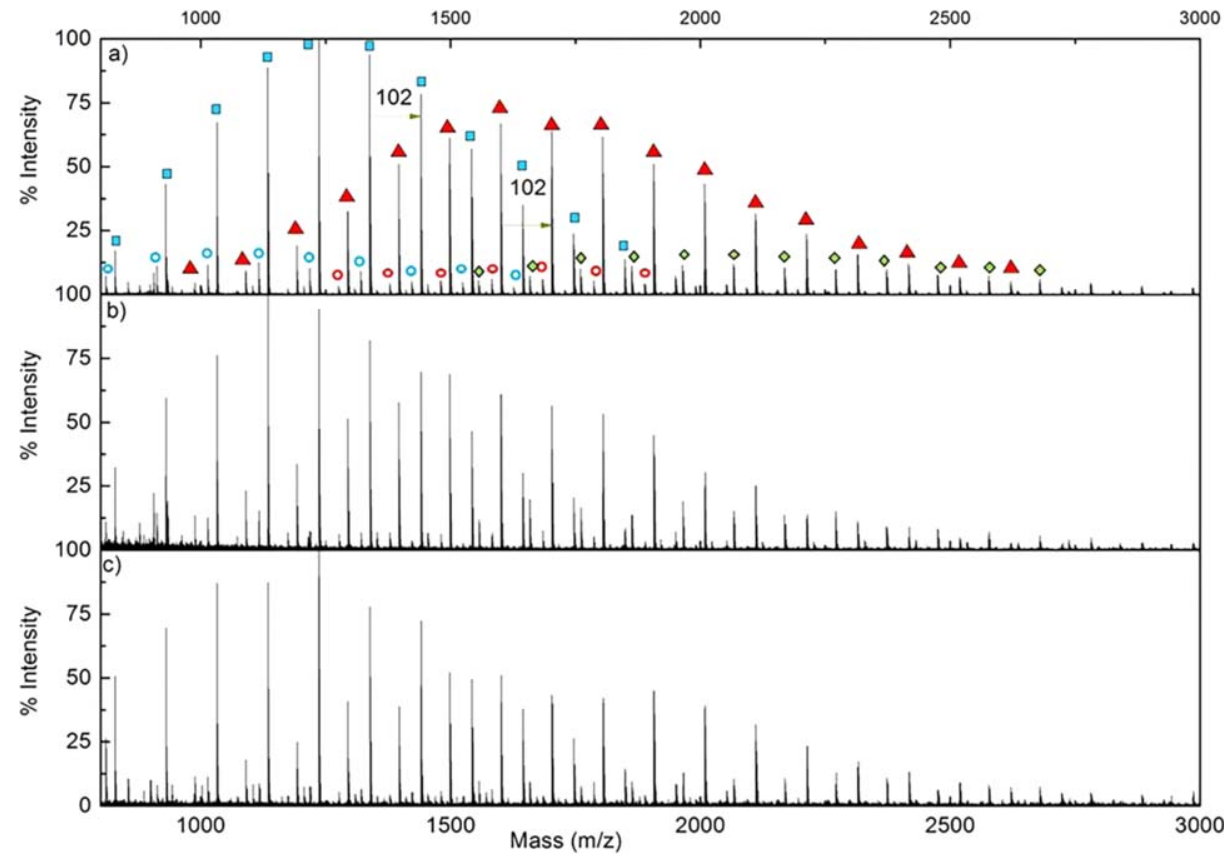

FIGURE 2 MALDI-MS spectra of polymer mixtures obtained for polymerization with fumaric acid. (a) Run 2, bulk, $90{ }^{\circ} \mathrm{C}, \mathrm{A} / \mathrm{l}=2.5$; (b) run 4 , bulk, $90{ }^{\circ} \mathrm{C}, \mathrm{A} / \mathrm{I}=2.5$, monomer added in multi-steps; (c) run 6 , bulk, $90{ }^{\circ} \mathrm{C}, \mathrm{A} / \mathrm{l}=0.5$. Molecular weight distribution for L-PTMC is indicated with blue squares and TMP-PTMC is indicated with red triangles. Side reactions due to intramolecular transesterification reactions are indicated in circles and decarboxylation is labeled with green rhombus. [Color figure can be viewed at wileyonlinelibrary. com]

When using a solvent in polymerization reactions, two effects play an important role. On the one hand, the solvent can help to solubilize the reagents leading to homogeneity and thus leading to faster reactions.

On the other hand, dilution can produce a delay in the polymerization, resulting in longer reaction times. Similar effects occur for reactions in bulk which can produce fast polymerizations, due to direct contact between the different reagents, or slow polymerizations, when poorly miscible components are used. Ideal polymerization conditions should permit to obtain a high monomer conversion (i.e., higher than $80 \%$ ) in relatively short reaction times, since longer reaction times might favor side reactions, even if a low $D$ is maintained.

For the reactions in toluene, the activation of the monomer by the acid is less efficient due to the dilution effect, what explains the longer reaction time needed. In what concerns $Ð$, GPC characterization showed low values for reactions, in bulk and solvent. However, a detailed study by MALDI revealed important information about its composition. Although in all experiments only one molecular mass distribution and low $Ð$ was observed in GPC (as reported in Table 1; a representative chromatogram is showed in Fig. S6†), the MALDI mass spectra (MS) of the polymers showed a mixture of several molecular weight distributions. Details of some of the MALDI MS are shown in Figure 2.

For both polymerization reactions with FA, with solvent (run 1) and in bulk [run 2, Fig. 2(a)], two main distributions were detected, due to the above mentioned competitive polymerization mechanisms: one corresponds to the polymer initiated by 1,3-propanediol via the ACE mechanism (76 (1,3-propanediol) + n·102 (molecular weight of TMC monomeric unit) +39 (potassium) $\mathrm{g} \mathrm{mol}^{-1}$ ) (blue squares) and the other corresponds with initiation by TMP by the AM mechanism (134 (TMP) + n.102+39 $\mathrm{g} \mathrm{mol}^{-1}$ ) (red triangles). Noteworthy, the distance between two consecutive peaks is $102 \mathrm{~m} / \mathrm{z}$, which corresponds with the molecular mass of the monomeric unit, confirming the insertion of TMC in both the L-PTMC and the TMP-PTMC polymer. Also some other minor distributions were detected and assigned to intramolecular transesterification or decarboxylation reactions [Fig. 2(a)]. The intramolecular transesterification reaction leads to the formation or cyclic species and was detected for both kind of polymers, L-PTMC and TMP-PTMC, fitting the molar masses with 134 (TMP) + n·102+39-18 $\left(\mathrm{H}_{2} \mathrm{O}\right) \mathrm{g} \mathrm{mol}^{-1}$ [open red circles in Fig. 2(a)] or 76 (1,3-propanediol) $+\mathrm{n} \cdot 102$ (TMC) +39 (potassium) $-18 \quad\left(\mathrm{H}_{2} \mathrm{O}\right) \mathrm{g}$ $\mathrm{mol}^{-1}$ [open blue circles in blue in Fig. 2(a)]. The decarboxylation distribution fitted with a molar mass of 134 $(\mathrm{TMP})+\mathrm{n} \cdot 102+39-44\left(\mathrm{CO}_{2}\right) \mathrm{g} \mathrm{mol}^{-1}$ [green rhombus in Fig. 2(a)].

When using TFA in solvent or bulk reactions (run 7-9 vs. run 13-15 in Table 1, respectively), high conversions were reached at similar reaction times, with slightly higher conversion and $D$ values when toluene was used. Contrary to FA, and due to the homogeneous catalytic character of TFA, in the latter case very similar results were obtained for the conversion of both types of reaction. For the bulk polymerizations at different 


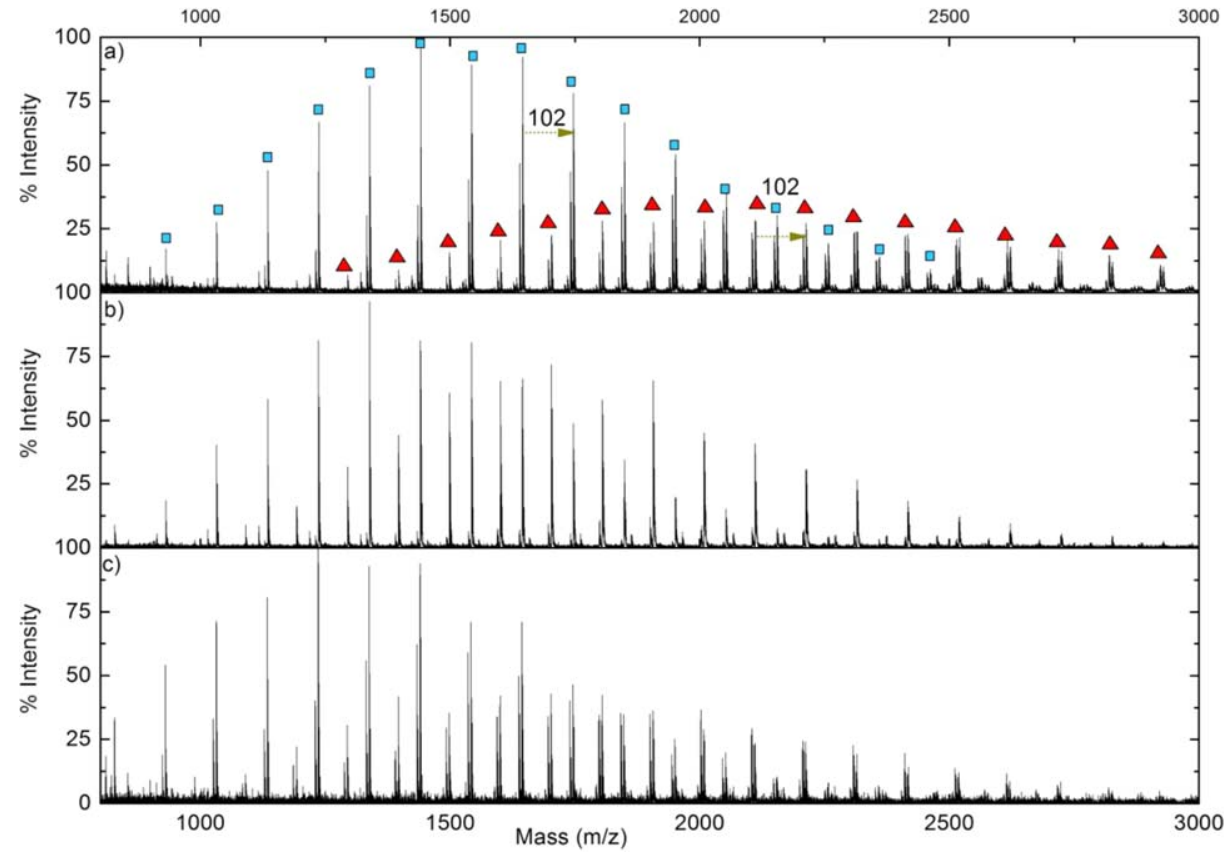

FIGURE 3 MALDI-MS spectra of polymer mixtures obtained for polymerization with trifluoroacetic acid. (a) Run 9 , toluene, $35^{\circ} \mathrm{C}, \mathrm{A} / \mathrm{l}=2.5$; (b) run 10 , toluene, $35^{\circ} \mathrm{C}, \mathrm{A} / \mathrm{I}=1$; (c) run 11 , toluene, $35^{\circ} \mathrm{C}, \mathrm{A} / \mathrm{I}=1$, with pre-distillation treatment. Molecular weight distribution for $\mathrm{L}-\mathrm{PTMC}$ is indicated with blue squares and TMP-PTMC is indicated with red triangles. [Color figure can be viewed at wileyonlinelibrary.com]

temperatures, $70{ }^{\circ} \mathrm{C}, 50{ }^{\circ} \mathrm{C}$, and $35^{\circ} \mathrm{C}$ (run $13-15$, respectively), similar MALDI MS results were obtained (Fig. S8†), showing the presence of two main polymer distributions attributed to the L-PTMC and the TMP-PTMC, as well as, other less intense distributions due to the side reactions, as for example intramolecular transesterification as assigned in Figure S8†.

For the polymerizations in toluene, however, the L-PTMC and TMP-PTMC were only detected when doing polymerizations at the lowest temperature, $35{ }^{\circ} \mathrm{C}$ [Fig. 3(a)]. At $70{ }^{\circ} \mathrm{C}$ and $50{ }^{\circ} \mathrm{C}$, both polymers were almost not present (Fig. S7†) and only small distributions attributed to products of side reactions could be detected.

The differences in the MALDI MS results for toluene and bulk polymerizations with TFA can be explained by the apolar reaction environment for the toluene polymerizations. This apolar environment renders the protonated intermediate monomer, formed after activation by the acid (see Scheme 1, right path) less stable, and therefore it reacts immediately, resulting in uncontrolled reactions. Hence, the combination of an apolar reaction medium with high temperatures directed the polymerizations toward side reactions, and no L-PTMC or TMP-PTMC polymers were obtained. These polymers were only observed in a majority when a sufficient stabilization of the intermediate protonated monomer in toluene was achieved, that is, by reducing the temperature to $35{ }^{\circ} \mathrm{C}$. The temperature effect for the polymerizations is further discussed in the following section.

\section{Effect of Temperature}

To study the effect of temperature variations, on the characteristics of the polymer products, experiments were first done with FA/bulk at $90{ }^{\circ} \mathrm{C}$ and at $100{ }^{\circ} \mathrm{C}$ (run 2-3 in Table 1 , respectively), following previous work on our group with these reaction conditions. ${ }^{37}$ The polymerization at higher temperature reached a slightly higher conversion after $48 \mathrm{~h}$, but both polymers showed a low $D$. The MALDI MS showed also in this case two main polymer populations for both temperatures, one for the TMP-PTMC and the other for the L-PTMC [Fig. 2(a) for run 2]. Hence, the temperature variations tested did not result in noticeable changes for the products of FA polymerizations. Being a heterogeneous catalyst, FA only activates the monomer at high temperature when the reaction is performed in bulk. ${ }^{23,37}$ Hence, an increase in the polymerization temperature did not result in any significant difference in the polymerization time or polymer characteristics, probably due to the similar activated-monomer availability in the reaction medium, which hardly changes with an increase in temperature of $10^{\circ} \mathrm{C}$.

When using TFA/bulk polymerizations (run 13-15), similar reaction times and MALDI MS results were obtained (Fig. S8†). An increase in temperature did not produce any change in reaction time or in the occurrence of side reactions, suggesting the same stability and reactivity of the activated monomer, independently of temperature.

Contrary to the results for the FA/bulk and TFA/bulk polymerizations, temperature variations had a large effect on the final polymer composition when polymerizations were done in TFA/toluene as catalyst (as discussed in the previous section). At the lowest polymerization temperature, $35{ }^{\circ} \mathrm{C}$ [run 9, Fig. $3(\mathrm{a})]$, the incidence of side reactions was reduced and L-PTMC and TMP-PTMC polymers were obtained as the main products. 
What is also notable is the low monomer conversion $(\approx 13 \%$ in $48 \mathrm{~h}$ ) obtained when the reaction was performed at room temperature, which underlines the importance of a reaction temperature compromise to achieve TMC polymerization when TFA is used as catalyst. The high acidity of TFA ( $\mathrm{pKa}=0.23$ ), combined with the high temperature and the apolar character of the reaction medium for toluene polymerizations, may lead to fast reaction of the protonated monomer intermediate resulting in non-controlled polymerizations toward side reactions. A reduction in temperature results in a more "controlled" polymerization, probably due to the higher stability of protonated monomer, directing polymerizations to the $\mathrm{AM}$ and ACE mechanisms, and resulting also in lower $\emptyset$.

Interestingly, when comparing polymerizations performed at the lowest temperature, $35^{\circ} \mathrm{C}$, in toluene or bulk (run 9 vs. run 15 respectively), very similar MALDI MS results were obtained, but less side reactions were present when the reaction was done in toluene [Fig. 3(a) vs. Fig. S8c†, zoom in details from 1790 to $1870 \mathrm{~m} / \mathrm{z}$ ]. This led us to use these experimental conditions for further synthesis optimization.

\section{Effect of Acid to Initiator Ratio}

Typically, when reducing the acid to initiator (A/I) ratio, lower polymerization rates are expected. In our work, by reducing the A/I molar ratio in FA polymerizations (run 2, 5, and 6 , respectively) no differences in $D$ or conversion were observed, the latter all being higher than $80 \%$ after 48 h. In these cases the L-PTMC and the TMP-PTMC were also detected in the MALDI MS [run 2, Fig. 2(a) and run 6, Fig. 2 (c)], but no significant differences were observed in terms of polymer composition, when decreasing the A/I ratio. The comparable polymerization rates observed indicate a similar acid availability to activate the monomer, independently from the A/I molar ratio feed, which can also explain the similarities in MALDI MS results. The same acid availability is a consequence of the heterogeneous character of FA in the reactions.

For TFA polymerizations, however, a reduction in A/I ratio from 2.5 (run 9), to 1 (run 10) to 0.5 (run 12) resulted in slower polymerization rates, with more time needed to reach conversions higher than $80 \%$. When looking at the respective MALDI MS data, "cleaner" mass spectra were obtained, showing the presence of the TMP-PTMC and L-PTMC polymers with no other residual molecular weight distributions, as shown in Figure 3(b). A decrease in A/I ratios for TFA, which is a homogeneous catalyst, yields less acid availability in the reaction media, and thus in less activation of the monomer, increasing the reaction times but resulting in more controlled reactions.

The composition of the obtained polymer mixture was quantified by ${ }^{1} \mathrm{H}$ NMR, by using the integrated values of the "c" protons of TMP-PTMC and the "g" protons in the polycarbonate chains for both polymers, as shown in Figure S3†. The molar composition calculated from the integrated signals is $68.5 \%$ of TMP-PTMC and $31.5 \%$ of L-PTMC, which indicates that with these optimized polymerization conditions we can obtain a polymer mixture composed mainly of a branched TMP-PTMC polymer. The full characterization of the polymer obtained in Run 10 is included in the supporting information, Figures $\mathrm{S} 3-\mathrm{S} 6+$, as a representative case.

For MSA polymerizations, decreasing the A/I ratios led to complete conversion after $2.5 \mathrm{~h}$ for all the reactions. Similar MALDI MS spectra as for the other reactions were obtained, and the L-PTMC and TMP-PTMC polymers, together with smaller molecular mass distributions, were identified (a representative spectrum is shown in Fig. S9†). However, a lower intensity for the TMP-PTMC polymer in the MALDI-MS spectra was detected, as compared with TFA polymerizations (Fig. 3), which may be related to the fact that MSA is a stronger homogeneous catalyst as compared with TFA. In general, although very fast and high conversions were reached, the polymerizations with MSA led to the highest $D$ values.

\section{Effect of Monomer Concentration: Monomer Addition in Multi-Steps}

Previously, Navarro et al. $^{25}$ reported a dependence of the final composition of the polymer products on the monomer concentration. According to these authors, by maintaining the monomer to initiator (M/I) molar ratio below 10 and doing the addition of the monomer in multiple steps, the polymer synthesis was forced in the direction to the AM mechanism. This multi-feed step, controlling the monomer concentration, has been effectively used for the controlled growth of linear polycarbonates by the AM mechanism only. $^{25}$

Following this work, we performed a polymerization with similar experimental conditions, that is, adding the monomer in several steps, and using FA as catalyst (run 4 in Table 1). Comparing the MALDI MS for the polymerization made with only one step [run 2, Fig. 2(a)] or in multiple steps [run 4, Fig. 2(b)], no differences were detected in our case. Again, the two main molecular mass distributions for L-PTMC and TMP-PTMC polymers were observed [Fig. 2(b)]. This result indicates that, for the experimental conditions tested, the monomer concentration had no effect on the kind of mechanism the polymerization follows.

\section{Effect of Monomer Purity}

ROP reactions are typically performed in very dry conditions to avoid parallel initiation by water molecules. Although we extensively dried all the reagents and the experimental setup before the polymerizations, it is still possible that a trace of water was present in the system. A consequence of the initiation by water, and the associated opening a TMC monomeric unit, would be the in situ formation of 1,3-propanediol which could explain the appearance of the linear polycarbonate in the polymer mixtures we discussed above.

To assess the origin of this linear polymer distribution, an experiment with an extremely pure, that is, exhaustively dried, monomer was done. To remove residual water as much as possible, a pre-distillation step was introduced into 
the polymerization system (run 11 in Table 1). TMC and TMP were initially dissolved in dry toluene. Thereafter, about $80 \%$ of the solvent was distilled under vacuum dragging all the possible residual water out of the reaction flask. TFA was then added to the TMP and TMC containing distilled solution, proceeding in a similar way as described in the Experimental section. The polymer obtained by this procedure showed again two molecular mass distributions in the MALDI MS [Fig. 3(c)] and confirms the origin of the linear polymer $n$ the occurrence of the ACE mechanism eliminating the possibility of initiation by residual water molecules.

\section{CONCLUSIONS}

Three acids, FA, TFA, and MSA, were effectively employed as CROP catalysts for TMC by using TMP as a tri-functional initiator. For all the acids and nearly all the polymerization conditions tried, at least two polymer distributions were detected by MALDI. These distributions consist of the TMPinitiated polymer, TMP-PTMC, and a linear polycarbonate, LPTMC, which are obtained via two competitive polymerization mechanisms, AM and AM/ACE. In some cases, also other polymer distributions were detected as a result of side reactions. With our study we could devise the optimal polymerization conditions, that is, the use of TFA as catalyst in toluene with equimolar acid to initiator ratios at $35{ }^{\circ} \mathrm{C}$, to avoid undesired side reactions. Under these conditions the polymer mixture contained about $68.5 \%$ of TMP-PTMC and $31.5 \%$ of the L-PTMC.

In this manuscript, we also found the importance of combining GPC with other characterization technique which can elucidate the molecular structure and polymer composition, being in this case MALDI an indispensable tool to detect the occurrence of these polymerization mechanisms when TMP was used as a tri-functional initiator.

The polymers prepared by this metal-free synthesis allowed the obtention of metal-free polymers with low $D$ value and well-known chemical structure and composition, which can be further used as polymeric matrix for coating preparation or in the biomedical or pharmacological field.

\section{ACKNOWLEDGMENTS}

The authors thank the Dutch Ministry of Economic Affairs, Agriculture and Innovation for funding via the IOP Self-Healing Materials program in the Netherlands (project \# SHM012044).

\section{REFERENCES AND NOTES}

1 N. De Vietro, L. Belforte, V. G. Lambertini, F. Fracassi, Appl. Surf. Sci. 2014, 307, 698-703.

2 H. D. Hwang, H. J. Kim, React. Funct. Polym. 2011, 71, 655665.

3 S. Nakano, Prog. Org. Coat. 1999, 35, 141-151.
4 E. Lay, D. S. Wuu, S. Y. Lo, R. H. Horng, H. F. Wei, L. Y. Jiang, H. U. Lee, Y. Y. Chang, Surf. Coat. Technol. 2011, 205, 4267-4273.

5 W. Chen, F. Meng, R. Cheng, C. Deng, J. Feijen, Z. Zhong, J. Control. Release 2014, 190, 398-414.

6 Y. Xu, X. Wu, X. Xie, Y. Zhong, R. Guidoin, Z. Zhang, Q. Fu, Polymer 2013, 54, 5363-5373.

7 K. Fukushima, Biomater. Sci. 2016, 4, 9-24.

8 F. Suriano, O. Coulembier, J. L. Hedrick, P. Dubois, Polym. Chem. 2011, 2, 528-533.

9 N. E. Kamber, W. Jeong, R. M. Waymouth, R. C. Pratt, B. G. G. Lohmeijer, J. L. Hedrick, Chem. Rev. 2007, 107, 5813-5840.

10 F. Nederberg, B. G. G. Lohmeijer, F. Leibfarth, R. C. Pratt, J. Choi, A. P. Dove, R. M. Waymouth, J. L. Hedrick, Biomacromolecules 2007, 8, 153-160.

$11 \mathrm{~S}$. Matsumura, K. Tsukada, K. Toshima, Macromolecules 1997, 30, 3122-3124.

12 H. R. Kricheldorf, R. Dunsing, A. S. I. Albet, Makromol. Chem. 1987, 188, 2453-2466.

13 A. C. Albertsson, R. K. Srivastava, Adv. Drug Deliv. Rev. 2008, 60, 1077-1093.

14 D. J. Darensbourg, P. Ganguly, D. Billodeaux, Macromolecules 2005, 38, 5406-5410.

15 C. Fliedel, S. Mameri, S. Dagorne, T. Aviles, Appl. Organomet. Chem. 2014, 28, 504-511.

16 D. M. Stevens, H. A. Watson, M. A. LeBlanc, R. Y. Wang, J. Chou, W. S. Bauer, E. Harth, Polym. Chem. 2013, 4, 2470-2474.

17 P. Kubisa, S. Penczek, Prog. Polym. Sci. 1999, 24, 14091437.

18 F. Sanda, H. Sanada, Y. Shibasaki, T. Endo, Macromolecules 2001, 35, 680-683.

19 T. Ariga, T. Takata, T. Endo, Macromolecules 1997, 30, 737744.

20 Y. Shibasaki, F. Sanda, T. Endo, Macromol. Rapid Commun. 1999, 20, 532-535.

21 A. Duda, Z. Florjanczyk, A. Hofman, S. Slomkowski, S. Penczek, Macromolecules 1990, 23, 1640-1646.

22 V. T. Lipik, L. K. Widjaja, S. S. Liow, M. J. M. Abadie, S. S. Venkatraman, Polym. Degrad. Stab. 2010, 95, 2596-2602.

23 T. Endo, Y. Shibasaki, F. Sanda. J. Polym. Sci. Part A: Polym. Chem. 2002, 40, 2190-2198.

24 J. M. Campos, M. R. Ribeiro, M. F. Ribeiro, A. Deffieux, F. Peruch, Macromol. Chem. Phys. 2013, 214, 85-93.

25 D. Delcroix, B. Martin-Vaca, D. Bourissou, C. Navarro, Macromolecules 2010, 43, 8828-8835.

26 J. Matsuo, S. Nakano, F. Sanda, T. Endo, J. Polym. Sci. Part A: Polym. Chem. 1998, 36, 2463-2471.

27 J. Jansen, M. J. Boerakker, J. Heuts, J. Feijen, D. W. Grijpma, J. Control. Release 2010, 147, 54-61.

28 B. van Bochove, J. J. Rongen, G. Hannink, T. G. van Tienen, P. Buma, D. W. Grijpma, Polym. Adv. Technol. 2015, 26, 14281432.

29 D. W. Grijpma, Q. Hou, J. Feijen, U.S. Patent 2012/0,322,953, 2012.

30 D. W. Grijpma, Q. Hou, J. Feijen, Biomaterials 2005, 26, 2795-2802.

31 P. Löwenhielm, H. Claesson, A. Hult, Macromol. Chem. Phys. 2004, 205, 1489-1496.

32 P. Lowenhielm, D. Nystrom, M. Johansson, A. Hult, Prog. Org. Coat. 2005, 54, 269-275. 
33 T. Saito, Y. Aizawa, K. Tajima, T. Isono, T. Satoh, Polym. Chem. 2015, 6, 4374-4384.

34 J. Liu, S. Cui, Z. Li, S. Xu, J. Xu, X. Pan, Y. Liu, H. Dong, H. Sun, K. Guo, Polym. Chem. 2016, 7, 5526-5535.

35 H. Wu, Y. Ji, Z. Li, X. Wang, O. Zhang, S. Cui, W. Wu, J. Liu, K. Guo, J. Polym. Sci. Part A: Polym. Chem. 2015, 53, 729-736,
36 X. He, Y. Ji, Y. Jin, S. Kan, H. Xia, J. Chen, B. Liang, H. Wu, K. Guo, Z. Li, J. Polym. Sci. Part A: Polym. Chem. 2014, 52, 1009-1019.

37 T. Dikić, W. Ming, P. C. Thüne, R. A. T. M. van Benthem, G. de With, J. Polym. Sci. Part A: Polym. Chem. 2008, 46, 218-227. 